\title{
Article
}

\section{Phthalate side side-chain structures and hydrolysis metabolism associated with steroidogenic effects in MLTC-1 Leydig cells}

Tian, Meiping, Zhang, Xi, Martin, Francis L, Wang, Heng, Zhang, Jie, Huang, Qingyu, Wang, Xiaofei and Shen, Heqing

Available at http://clok.uclan.ac.uk/28102/

Tian, Meiping, Zhang, Xi, Martin, Francis L ORCID: 0000-0001-8562-4944, Wang, Heng, Zhang, Jie, Huang, Qingyu, Wang, Xiaofei and Shen, Heqing (2019) Phthalate side side-chain structures and hydrolysis metabolism associated with steroidogenic effects in MLTC-1 Leydig cells. Toxicology Letters . ISSN 0378-4274

It is advisable to refer to the publisher's version if you intend to cite from the work. http://dx.doi.org/10.1016/j.toxlet.2019.03.013

For more information about UCLan's research in this area go to http://www.uclan.ac.uk/researchgroups/ and search for <name of research Group>.

For information about Research generally at UCLan please go to http://www.uclan.ac.uk/research/

All outputs in CLoK are protected by Intellectual Property Rights law, including Copyright law. Copyright, IPR and Moral Rights for the works on this site are retained by the individual authors and/or other copyright owners. Terms and conditions for use of this material are defined in the policies page. 


\section{Accepted Manuscript}

Title: Phthalate side-chain structures and hydrolysis metabolism associated with steroidogenic effects in MLTC-1

Leydig cells

Authors: Meiping Tian, Xi Zhang, Liangpo Liu, Francis L. Martin, Heng Wang, Jie Zhang, Qingyu Huang, Xiaofei Wang,

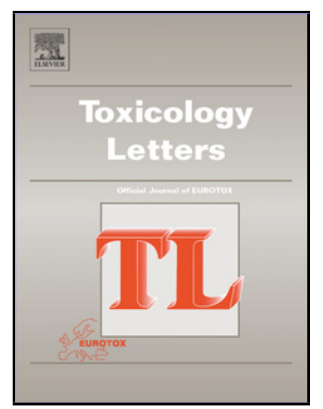
Heqing Shen

PII:

DOI:

Reference:

S0378-4274(18)32015-0 https://doi.org/10.1016/j.toxlet.2019.03.013

To appear in: $\quad$ Toxicology Letters

Received date: $\quad 13$ October 2018

Revised date: $\quad 5$ February 2019

Accepted date: $\quad 28$ March 2019

Please cite this article as: Tian M, Zhang X, Liu L, Martin FL, Wang H, Zhang J, Huang Q, Wang X, Shen H, Phthalate side-chain structures and hydrolysis metabolism associated with steroidogenic effects in MLTC-1 Leydig cells, Toxicology Letters (2019), https://doi.org/10.1016/j.toxlet.2019.03.013

This is a PDF file of an unedited manuscript that has been accepted for publication. As a service to our customers we are providing this early version of the manuscript. The manuscript will undergo copyediting, typesetting, and review of the resulting proof before it is published in its final form. Please note that during the production process errors may be discovered which could affect the content, and all legal disclaimers that apply to the journal pertain. 


\section{Phthalate side-chain structures and hydrolysis metabolism associated with steroidogenic effects in MLTC-1 Leydig cells}

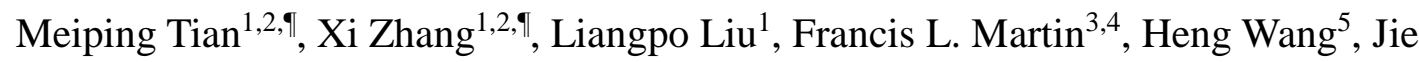
Zhang $^{1}$, Qingyu Huang ${ }^{1}$, Xiaofei Wang ${ }^{1}$, Heqing Shen ${ }^{1}$

${ }^{1}$ Key Laboratory of Urban Environment and Health, Institute of Urban Environment, Chinese Academy of Sciences, Xiamen 361021, China

${ }^{2}$ University of Chinese Academy of Science, Beijing 100049, China

${ }^{3}$ Department of Gynaecology \& Obstetrics, Lancashire Teaching Hospitals NHS

Trust, Fulwood, Preston PR2 9HT, UK

${ }^{4}$ Biocel Ltd, Hull, Yorkshire, UK

${ }^{5}$ Key Laboratory of Health Risk Factors for Seafood of Zhejiang Province, Zhoushan Municipal Center for Disease Control and Prevention, Zhejiang Zhoushan, 316021, China

IIMeiping Tian and Xi Zhang contributed equally to this work.

\section{Corresponding Authors:}

Meiping Tian, Institute of Urban Environment, Chinese Academy of Sciences, Key Laboratory of Urban Environment and Health, 1799 Jimei Road, Xiamen 361021, China; Telephone/Fax: (86)-592-6190523; E-mail: mptian@iue.ac.cn Heqing Shen, Institute of Urban Environment, Chinese Academy of Sciences, Key Laboratory of Urban Environment and Health, 1799 Jimei Road, Xiamen 361021, China; Telephone/Fax: (86)-592-6190771; E-mail: hqshen@iue.ac.cn

\section{Highlights}

$>\quad$ Phthalates' side-chain structures associated with hydrolysis metabolism.

$>\quad$ Phthalate metabolites appear to exert different effects at an endocrine level compared to parent compounds. 
Hydrolysis metabolism reversed DBP-induced anti-androgenic effects into the androgenic effects.

\begin{abstract}
Although it is well acknowledged that the anti-androgenic phthalate diesters can be readily hydrolysed into their monoester counterparts, their metabolites' toxicology remains obscure. Herein, we tested the hypothesis that hydrolysis of one of the two ester bonds can mediate phthalate diesters' potential endocrine effects in MLTC-1 Leydig cells, in line with their ability to disrupt androgen secretion in humans. Five diesters (DMP, DEP, DBP, DBzP and DEHP) and five monoesters (MMP, MEP, MBP, $\mathrm{MBzP}$ and MEHP) phthalates as mixtures or individually were applied to cell lines to investigate differences in phthalates' hydrolysis associated with varying side-chain structures and steroidogenic effects. Short-chain diesters DMP, DEP and DBP are more readily hydrolysed compared to the long-chain DEHP, while aromatic alkyl chain DBzP cannot be metabolized completely in vitro. When the hydrolysis processes are interrupted, the diester phthalates' steroidogenic effects can be influenced via regulating related steroidogenic pathway genes. With 10 to $100 \mu \mathrm{M}$ treatment exposures, androgenic effects were observed only with DMP or DEP but not for MMP or MEP; while the phthalate diesters DBP, DBzP or DEHP generally exhibited more complex steroidogenic effects than their corresponding monoester counterparts (i.e., biphasic androgen and anti-androgen effects for diesters but monotonic androgen effects for monoesters were observed). DBP elicited hydrolysis-related steroidogenic modulation, in which the anti-androgenic effects of diester DBP reversed into the androgenic effects of monoester MBP at $100 \mu \mathrm{M}$. Phthalate metabolites appear to exert different effects at an endocrine level compared to parent compounds, and deeper insights into how the hydrolytic process is related to this alternating toxicity would improve our understanding of a risk assessment for these widespread contaminants in male reproduction.
\end{abstract}

Keywords: Androgen, Hydrolysis metabolism, MLTC-1 cells, Phthalate, Steroidogenesis 


\section{Introduction}

Phthalates are diesters of 1, 2-benzenedicarboxylic acid (o-phthalic acid) and their molecular structures contain one benzene ring and two esters. The two hydrocarbon chains with various structures confer phthalates with their various physical properties; the chains are straight or branching alkanols with increasing carbons from methanol to tridecanol (Fernández et al. 2012; Park et al. 2014). These unique physicochemical properties mean that phthalates are ideally suited for applications in industrial and consumer products, including plasticizers, food packaging materials, toys, personal care products and medical devices (Shea 2003; Mankidy et al. 2013). Phthalates are not chemically bound to products, and hence, they can easily migrate from these matrices into the environment and human body via a variety of routes (Blanchard et al. 2014; Swan 2008). Generally, humans are exposed to phthalates via oral, inhalation, dermal and intravenous routes (Hauser and Calafat 2005).

As endocrine disrupting chemicals, phthalates are generally thought to be anti-androgens and are suspected to generate adverse effects on the male reproductive system (Moore et al. 2001; Mankidy et al. 2013). Phthalates' alkanol moieties are associated with their anti-androgen potency. In foetal male rat models, phthalates exhibit a structure-specific inhibition to testosterone production in testis: diphenyl ortho-phthalate (DPP) is more potent than butyl benzyl phthalate (BBP), dibutyl phthalate (DBP) and diisobutyl phthalate (DIBP), while short-chain diethyl phthalate (DEP) and dimethyl phthalate (DMP) have no observable effects (Clewell et al. 2010; 
Howdeshell et al. 2008).

Post-oral uptake, lipophilic phthalate diesters can be initially transformed via phase I hydrolysis. In mammals, phthalate diesters are rapidly hydrolysed by lipases or esterases to generate phthalate monoesters; subsequently, some median to long side-chain monoesters will be further metabolized by cytochrome P450s (CYPs) via $\beta$-oxidation. Then these metabolites are phase II conjugated before they are excreted via the urine (Calafat et al. 2006; Frederiksen et al. 2007). Esterases involved in phthalates' phase I hydrolysis can be found in the small intestine, colon, kidney, liver, heart brain and testis (Harris et al. 2016; Xu et al. 2002).

Although not always the case, metabolism generally protects the organism against xenobiotic-induced toxicity. A recent study indicates that phthalate diesters are more toxic than monoesters (Xu et al. 2016), while another study suggests that phthalates in their diester or glucuronidated metabolic forms are non-toxic (Stein et al. 2013), whilst another suggests that there is no difference in toxicity profile between monoester and glucuronidated metabolites (Katsikantami et al. 2016). Therefore, although phthalates' metabolism has been widely characterized, the association of these metabolic processes and endocrine effect alterations remains under debate.

Testicular Leydig cells are the primary source of steroid hormone in the male, and steroid hormone production starts with cholesterol, which is converted into one of the important intermediates of androstenedione (ASD) before production of the end-product sex hormone, testosterone (Bochud et al. 2018). Moreover, a previous study confirmed that in vitro testicular cells can hydrolyse phthalate diesters into 
monoesters forms (Harris et al., 2016); mouse Leydig tumour cells (MLTC-1) are a useful tool in the assessment of effects on steroidogenesis (Svechnikov et al. 2016).

Herein, phthalate hydrolysis interference experiments were employed to examine the possible associations of hydrolytic processes with steroidogenesis, and the phthalates side-chain structures associated hydrolytic metabolism in MLTC-1 Leydig cells. The aim of this study is to elucidate the association between phthalate hydrolysis and their ability to induce endocrine disrupting alterations. The results will improve our understanding of the hydrolytic processes related to toxic changes of these widespread male reproductive contaminants.

\section{Materials and methods}

\subsection{Chemicals and reagents}

The following phthalate diesters: dimethyl phthalate (DMP), diethyl phthalate (DEP), dibutyl phthalate (DBP), di-(2-ethylhexyl) phthalate (DEHP) and dibenzyl phthalate (DBzP) were purchased from TCI (Tokyo Chemical Industry Europe, Belgium, Brussels). The $\mathrm{C}_{13}$ - and $\mathrm{C}_{12}$-phthalate monoesters: monomethyl phthalate (MMP), monoethyl phthalate (MEP), monobutyl phthalate (MBP), mono-2-ethylhexyl phthalate (MEHP) and monobenzyl phthalate (MBzP) were purchased from CIL (Cambridge Isotope Laboratory Ins, USA). The selected phthalates' structures are shown in Table S1 [see Supplementary Information (SI)]. Dimethyl sulfoxide (DMSO), esterase, Escherichia coli ß-glucuronididase and constitutive androstane receptor $(\mathrm{CAR})$ inhibitor 
1-(2-Chlorophenyl)-N-methyl-N-(1-methylpropyl)-3-isoquinolinecarboxamide (PK 11195) were purchased from Sigma-Aldrich Chemical Co. (St. Louis, Mo. USA). Human chorionic gonadotrophin (hCG) was obtained from PROSPECT (Ness-Ziona, Israel). The steroid hormone standards of androstenedione (ASD), testosterone and 17-OH progesterone (17-OHP) were purchased from Dr. Ehrenstorfer GmbH (Augsburg, Germany). The isotopes D3-testosterone and D3-17-OHP were purchased from Cerilliant (Promochem, Wesel, Germany). Oasis HLB solid-phase extraction (SPE) cartridges (60 mg, $3 \mathrm{~mL}$ ) were purchased from Waters Corporation (Milford, MA). The Carboxylesterase (Ces) Activity Assay Kit was obtained from Nanjing Jiancheng Bioengineering Institute (Nanjing, China).

\subsection{Cell culture and phthalate treatment}

The MLTC-1 cell line was obtained from the Cell Institute of Shanghai (Shanghai, China) and cultured in RPMI-1640 medium (Gibco BRL, Grand Island, NY) supplemented with 100 unit $/ \mathrm{mL}$ penicillin, $100 \mathrm{unit} / \mathrm{mL}$ streptomycin and $10 \%$ (v/v) foetal bovine serum (Hyclone, USA). The cells were grown at $37^{\circ} \mathrm{C}$ with $5 \%$ $\mathrm{CO}_{2}$ in a humidified incubator (SANYO, Japan). The MLTC-1 cells were seeded in 10-cm petri dishes and cultured for $48 \mathrm{~h}$ prior to further treatment. Phthalates were dissolved in DMSO. Cell viability was evaluated by the MTT proliferation assay (see SI Figure S1) to ensure that non-cytotoxic concentrations were employed for the following phthalates exposure experiments.

The first set of experiments was designed to assess the hydrolysis metabolism profiles of phthalate diesters and the different endocrine disrupting effects between 
phthalate diesters and monoesters mixture exposure. Referencing the MTT results and previous studies reporting the general Chinese male population maximum exposure levels (0.03 to $0.48 \mu \mathrm{M})$ (Tian et al., 2018) and the general German male population (0.32 to $3.70 \mu \mathrm{M})$ (Koch et al., 2003), MLTC-1 cells were exposed to $0.1 \%(\mathrm{v} / \mathrm{v})$

DMSO vehicle control, four levels of equimolar diester phthalate mixtures (i.e., 0.1, 1, 10 or $100 \mu \mathrm{M}$ of DMP, DEP, DBP, DBzP and DEHP in mixtures) and four levels of equimolar monoester phthalate mixtures (i.e., $0.1,1,10$ or $100 \mu \mathrm{M}$ of MMP, MEP, MBP, MBzP and MEHP in mixtures).

The second set of experiments was designed to assess how the endocrine disrupting effect of high-dose phthalate diesters are modified when facilitating diesters hydrolysis by the extracellular esterase. MLTC-1 cells were exposed to DMSO $0.1 \%(\mathrm{v} / \mathrm{v})$ vehicle control, $100 \mu \mathrm{M}$ of equimolar phthalate diesters mixture (100 $\mu \mathrm{M}$ of DMP, DEP, DBP, DBzP or DEHP in mixtures), $100 \mu \mathrm{M}$ of equimolar phthalate monoesters mixture (100 $\mu \mathrm{M}$ of MMP, MEP, MBP, MBzP or MEHP in mixtures) and $100 \mu \mathrm{M}$ of phthalate diesters mixture plus carboxylic ester hydrolase (15 units; Sigma-Aldrich, USA) to facilitate phthalate diesters hydrolysis.

The third set of experiments was designed to assess how the endocrine disrupting effect of low-dose phthalate diesters are modified when diesters hydrolysis is inhibited by the intracellular carboxylesterase inhibitor. Carboxylesterase (Ces2a) gene expression and enzyme activity can be inhibited when the activity of CAR is inhibited by the antagonist PK 11195. In this set of experiments, MLTC-1 cells were exposed to $0.1 \%$ (v/v) DMSO vehicle control, PK $11195(5 \mu \mathrm{M}), 10 \mu \mathrm{M}$ of equimolar 
phthalate diesters mixture (10 $\mu \mathrm{M}$ of DMP, DEP, DBP, DBzP or DEHP in mixture), and $10 \mu \mathrm{M}$ of equimolar phthalate diesters mixture plus PK $11195(5 \mu \mathrm{M})$.

The last set of experiments was designed to assess the different endocrine disrupting effects between individual exposures of phthalate diesters and monoesters. MLTC-1 cells were exposed to $0.1 \%$ (v/v) DMSO vehicle control, the individual phthalate diesters (10 or $100 \mu \mathrm{M}$ of DMP, DEP, DBP, DBzP or DEHP, respectively) and the individual monoesters ( 1 or $10 \mu \mathrm{M}$ of MMP, MEP, MBP, MBzP or MEHP, respectively).

After 48-h exposure, the culture medium was collected for phthalate metabolites analysis, and the cells were washed with $4 \mathrm{ml}$ PBS and serum-free medium towards assessing steroidogenic functions. The cells were stimulated with hCG $(0.1 \mathrm{U} / \mathrm{ml})$ for $4 \mathrm{~h}$ in serum-free medium with $0.1 \%$ BSA. The medium was collected for $17-\mathrm{OHP}$, ASD and testosterone determination, and cells were either prepared for RNA extraction or gene expression analysis or for carboxylesterase activity determination.

\subsection{RNA extraction and real-time RT-PCR analysis}

To determine mRNA expression alterations, a quantitative real-time PCR assay was performed. Total RNA was extracted from cells using a RNA extraction kit (Promega, USA) following the manufacturer's protocol. Extracted RNA samples were stored at $-80^{\circ} \mathrm{C}$ for subsequent analysis. The NanoDrop spectrophotometer (NanoDrop Technologies Inc., USA) was used to measure RNA concentration and purity. Reverse transcription of cDNA synthesis was performed using oligo dT primer, in which $1 \mu \mathrm{g}$ total RNA using PrimeScript ${ }^{\circledR}$ RT reagent Kit together with gDNA Eraser cDNA 
synthesis Kits (Takara, Japan) were applied. Real-time PCR was carried in a final volume of $20 \mu \mathrm{L}$ and performed in triplicate using SYBR Green Master Mix reagents (Roche, USA) in a LC 480 system (Roche Applied Science, Germany) according to the manufacturer's protocol. Primer sets and product sizes used for amplification PCR analysis are shown as Table S2 (see SI). The conditions for quantitative PCR were as follows: $95^{\circ} \mathrm{C}$ for $10 \mathrm{~min}$ followed by 40 cycles at $95^{\circ} \mathrm{C}$ for $15 \mathrm{~s}$, and $60^{\circ} \mathrm{C}$ for $30 \mathrm{~s}$. Gene expression levels were normalized to GAPDH expression levels. Three replicates of quantitative PCR were performed for each sample. Three replicates for each dose of phthalates exposure were performed. The fold-changes of the tested genes were analysed by the $2^{-\Delta \Delta \mathrm{Ct}}$ method.

\subsection{Carboxylesterase activity assay}

Carboxylesterase activity was assessed using a commercial kit from the Nanjing Jiancheng Bioengineering Institute (Nanjing, China). In brief, spectrophotometric recordings of carboxylesterase activity measured in a previous study (Zhu and $\mathrm{He}$ $2000)$ with the modification of using $\alpha$-naphthyl acetate $(\alpha-\mathrm{NA})$ as substrate were used as standards. Concentration changes of the substrate's hydrolysis product were measured at $\lambda=450 \mathrm{~nm}$ on a SpectraMAX190 microplate reader and quantified by SOFTmax software (Molecular Devices, Sunnyvale, CA). Non-enzymatic controls (0.01 M Tris- $\mathrm{HCl}$ buffer, $\mathrm{pH}$ 7.4) were also assayed. Three biological and three technical replicates were performed. For estimation of protein, the BCA protein assay kit (Thermo Fisher Scientific, USA) was employed. The specific activity of carboxylesterase is defined as the increased absorption value per minute per milligram 
of cell protein, and the activity of carboxylesterase was expressed as units of enzyme activity of carboxylesterase per milligram protein (U/mg protein).

\subsection{Determination of phthalates metabolites and steroid hormones in cell culture} medium

Phthalate metabolites in cell culture medium were detected using a published method for isotope dilution liquid chromatography-electrospray-ionization coupled with tandem mass spectrometry (LC-ESI-MS/MS; Applied Biosystems/MDS SCIEX, Singapore). Five hydrolysed phthalate metabolites (i.e., MMP, MEP, MBP, MBzP and MEHP) were determined. Briefly, phthalate metabolites in cell medium were initially de-conjugated using Escherichia coli $\beta$-glucuronididase, then purified through a SPE cartridge, and finally analysed by an isotope dilution method using LC-ESI-MS/MS. Two method blanks, two quality control samples (sample spiked with standards) and two sets of standards were also run together with the unknown samples in each analytical batch. The contents of the total species (i.e., the conjugated monoester phthalates plus their free forms) were calculated by modifying our published method (Liu et al. 2012; Tian et al. 2018).

The steroid hormones 17-OHP, ASD and testosterone were detected in the cell culture medium by LC-ESI-MS/MS (Tian et al. 2018). In brief, each sample of $1 \mathrm{~mL}$ medium was diluted with $3 \mathrm{~mL}$ of ammonium acetate buffer $(1 \mathrm{~mol} / \mathrm{L})$, and $20 \mu \mathrm{L}$ of $100 \mathrm{ng} / \mathrm{mL}$ D3-testosterone and D3-17-OHP were added as internal standard (ASD was semi-quantified). Then the diluted samples were extracted by adding $3 \mathrm{~mL}$ ethyl acetate and vortexed vigorously for $15 \mathrm{sec}$ in a glass tube. The liquid-liquid extraction 
was repeated 3 times. Then the subsequent phase separation was achieved by centrifugation at $1500 \mathrm{rpm}$ for $10 \mathrm{~min}$. The organic phase was transferred into another glass tube with a Pasteur pipette. The three times extract was combined and washed with $5 \mathrm{~mL}$ water, then the combined extracts were evaporated under a gentle stream of nitrogen gas at $40^{\circ} \mathrm{C}$. The residual was reconstituted with $200 \mu \mathrm{L}$ of methanol/water (50:50, v:v) by vortexing vigorously for $15 \mathrm{sec}$ and then transferring into a HPLC vial. The sample was stored at $-20^{\circ} \mathrm{C}$ until LC-MS-MS analysis. Target compounds were separated by LC-20A prominence (Shimadzu, Kyoto, Japan) high-performance liquid chromatography (HPLC) equipped with a separation column (Hypersil GOLD ${ }^{\mathrm{TM}} \mathrm{aQ}, 100 \mathrm{~mm} \times 2.1 \mathrm{~mm}$, internal diameter $1.9 \mu \mathrm{m}$ particle size). The mobile phase A was water and phase B was methanol. Table S3 (see SI) shows the HPLC gradient program. The mobile phase flow rate was set at $0.30 \mathrm{~mL} / \mathrm{min}$, and the column temperature was set at $30^{\circ} \mathrm{C}$. The total run time for each injection was 12 min and the injection volume were $20 \mu \mathrm{L}$. The target compounds were analysed using LC-ESI-MS/MS. ESI-MS/MS was performed at unit mass resolution in multiple reaction monitoring $(\mathrm{MRM})$ under positive ion mode with the following parameters: source temperature (TEM) $450^{\circ} \mathrm{C}$, ion spray voltage (IS) $3000 \mathrm{~V}$, sheath gas pressure, 40 psi. Optimization of compound-dependent MS/MS parameters was performed with steroid hormone standards via direct injection into the mass spectrometer using a syringe pump at a flow rate of $10 \mu \mathrm{L} / \mathrm{min}$. Optimal settings for compound-dependent MS/MS parameters are shown in Table S4 (see SI). Two quality control samples and two sets of standards were analysed together with unknown samples in each analytical 
batch (see SI Table S5).

\subsection{Statistical analysis}

We performed data analysis using SPSS statistical software (SPSS Inc.). The measured continuous variables of gene expression, carboxylesterase activity, phthalate hydrolysis ratio and steroid hormone levels were presented as mean with their standard errors of mean (mean \pm SEM). Data were subjected to analysis by one-way ANOVA followed by Tukey's multiple comparison testing to identify significant differences between groups when $\geq 3$ groups were calculated. For all the tests, $p$-values $<0.05$ were considered as significant criteria.

\section{Results}

\subsection{Hydrolysis metabolism associated with phthalates endocrine disrupting} effects

Hydrolytic activity of MLTC-1 cells towards phthalate diesters were examined (Figure 1A). The hydrolytic activity of MLTC-1 cells varied depending on the phthalate structure; relatively weak hydrolytic activity was observed towards phthalates having longer or bulky alkyl side chains on the alcohol ester moiety, while DBzP with two bulky benzyl chains cannot be hydrolysed into MBzP in MLTC-1 cells at any concentration. The hydrolysis metabolism ratio declined linearly with an increasing level of phthalate diesters exposure. MLTC-1 cells exposed to low-dose (0.1-1 $\mu \mathrm{M})$ phthalate diesters (except DBzP), there was almost total hydrolysis to corresponding phthalate monoesters, while hardly any level was hydrolysed following 
high-dose $(100 \mu \mathrm{M})$ exposure. The endocrine disrupting potency and relevant steroidogenic pathway gene response between mixed phthalate diesters and monoesters were also investigated (Figure 1B-F, see SI Table S6). At low-dose exposure $(0.1-10 \mu \mathrm{M})$, phthalate diesters and monoesters exhibited androgenic effects (stimulating the production of ASD, 17-OHP and testosterone). At high-dose exposure $(100 \mu \mathrm{M})$, phthalate diesters exerted anti-androgenic effects (inhibiting the production of ASD, 17-OHP and testosterone) because of their incomplete hydrolysis, while phthalate monoesters still exhibited androgenic effects (stimulating the production of ASD). Meanwhile, steroidogenic pathway gene expression was involved in phthalates-induced endocrine disrupting effects but exhibited a less than obvious dose-response trend due to the mixture exposure (Figure 1B and C).

\subsection{Extracellular esterase-enhanced phthalate diesters' hydrolysis and}

\section{attenuation of their anti-androgenic effects}

In order to confirm the hypothesis that hydrolytic metabolism can attenuate phthalates diester' anti-androgenic effects, external addition of esterase was conducted at $100 \mu \mathrm{M}$ mixed dose, at which the anti-androgenic action for all three steroids was apparent (i.e., inhibited). With the $100 \mu \mathrm{M}$ mixture exposure, extracellular esterase significantly elevates the hydrolysis of DMP (from $19.30 \%$ to $59.20 \%, p<0.001$ ), DEP (from $6.71 \%$ to $34.95 \%, p<0.001$ ) and DBP (from $6.14 \%$ to $64.75 \%, p<0.001$ ); the change was not significant for DEHP (from $1.61 \%$ to $1.74 \%, p=0.92$ ) while no change is observed for DBzP (Figure 2A). When extracellular esterase was added in the $100 \mu \mathrm{M}$ diester mixture group, the diester anti-androgenic effects (i.e., decreased 
production of 17-OHP, ASD and testosterone) is completely reversed to androgenic effects (i.e., stimulating production of 17-OHP, ASD and testosterone); the observed androgenic action is similar to the mode of action of phthalates monoester mixture (Figure 2B-E). All selected androgen synthesis pathway genes except $17 \beta$-HSD (responsible for the ultimate conversion of ASD into testosterone) were inhibited at $100 \mu \mathrm{M}$ mixture diester phthalate (CYP17 $\alpha$ exhibiting greatest level of down-regulation) and suggests a total anti-androgenic effect on all steroids, while $C Y P 17 \alpha, P 450 S C C$ and $3 \beta-H S D$ gene expression are significantly increased with extracellular esterase co-exposure (Figure 2B, see SI Table S7). These data show that extracellular esterase significantly facilitates mixed diesters' hydrolysis, then attenuates anti-androgenic effects via modification of the steroidogenesis pathway gene expression profile. When compared to mixture monoesters in $100 \mu \mathrm{M}$ group, the down-regulation of $S R-B 1$ and $S t A R$ in $100 \mathrm{D}^{+}(100 \mu \mathrm{M}$ of phthalate diesters mixture plus carboxylic ester hydrolase) group relates the major diester residuals of DEHP and DBzP, which are hard to metabolize by extracellular esterase in cell lines (Figure 2B).

\subsection{Inhibited intracellular carboxylesterase associated with reduced hydrolysis and enhanced activity of diester phthalates}

In order to confirm phthalate diester hydrolysis-associated toxicological changes, carboxylesterase (Ces) intervention experiments were conducted. Ces2a is an enzyme coded by Ces that is regulated by nuclear orphan constitutive active receptor (CAR) and can catalyse intracellular phthalate diester hydrolysis. Ten $\mu \mathrm{M}$ mixture phthalate diesters were set because this exposure exhibits both the hydrolysis margin (Figure 
1A) and apparent effects on steroids. The results show PK 11195 is a CAR antagonist and can consequently significantly inhibit CAR-regulated Ces $2 a$ expression and enzyme activity in MLTC-1 cells (Figure 3A-B, see SI Table S8). When MLTC-1 cells are co-exposed with a mixture of phthalate diesters and PK 11195, DMP and DEP hydrolysis are significantly inhibited (Figure $3 \mathrm{C}$ ). Slight androgenic effects (Figure 1D-F and 3E-G) are observed in $10 \mu \mathrm{M}$ phthalate diesters mixture treatment, while significant anti-androgenic effects are observed when the phthalate diesters mixture is co-exposed with PK 11195 groups (decreased production of testosterone, $P$ $<0.05$ ) (Figure $3 \mathrm{G}$ ). Meanwhile, steroidogenesis pathway gene $3 \beta-H S D$ expression is significantly down-regulated in $10 \mu \mathrm{M}$ phthalate diesters mixture groups following

PK 11195 exposure $(P<0.05)$ (Figure 3D, see SI Table S8). The results show that PK 11195 induced inhibition of hydrolysis has a greater effect on $S R-B 1$ than other genes, which may then be transferred to down-stream genes such as StAR and $3 \beta-H S D$ (see SI Table S8).

\subsection{Individual different endocrine disrupting effects of phthalate in MLTC-1}

In order to directly compare the different effects of phthalate monoester with diester and distinguish structure-dependent toxicities, individual phthalate exposure experiments were conducted (Figure 4). In low-dose $(10 \mu \mathrm{M})$ experiments, the phthalate diesters of DEP, DBP, DBzP and DEHP and monoester phthalate MEHP tend to exert androgenic effects (i.e., increase production of ASD and 17-OHP); no significant effect was observed for other phthalates (Figure 4). In high-dose (100 $\mu \mathrm{M})$ treatments, DMP but not MMP exhibits stimulation of ASD production, and DEP but 
not MEP exhibits androgenic effects (induced production of 17-OHP, ASD and testosterone). DBP exhibits anti-androgenic effects (inhibits production of 17-OHP, ASD and testosterone), while MBP shows androgenic effects (stimulates synthesis of ASD and testosterone) at $100 \mu \mathrm{M}$ doses. DBzP inhibits 17-OHP and testosterone secretion, while both DBzP and MBzP have stimulant effects on ASD production and MBzP-induced testosterone secretion; DEHP inhibits 17-OHP, while DEHP stimulates ASD and MEHP stimulates 17-OHP and testosterone. In summary, if any effect tendencies arise from diester DMP or DEP or monoester MBP, MBzP and MEHP at 10 to $100 \mu \mathrm{M}$, they are stimulating; for other phthalates the effects are inhibitory for 17-OHP and testosterone at $100 \mu \mathrm{M}$ but stimulating for ASD at both 10 and $100 \mu \mathrm{M}$ doses.

Following treatment with phthalate monoesters, genes are up-regulated or unchanged with the two pathway terminal genes exhibiting the highest fold-changes (see SI Table S9; the down-regulated CYP17 $\alpha$ for $100 \mu \mathrm{M}$ MBP is apparent but not significant when compared to the DMSO control). When treated with low-dose phthalate diesters, genes are up-regulated or unchanged in their expression but with a generally reduced alteration when compared to $10 \mu \mathrm{M}$ monoester counterparts (see SI Table S9). The pathway initiation $S R-B 1$ gene encodes an integral membrane protein scavenger receptor class B and leads the up-regulation of androgenic effects, which may initially be the response to the androgenic action of phthalates. When all three steroids are inhibited (100 $\mu \mathrm{M}$ diester mixture or DBP treatments), StAR, CYP17 $\alpha$ and $P 450 S C C$ are all down-regulated and $C Y P 17 \alpha$ is minimally down-regulated. However, 
while incomplete steroid inhibition (ASD stimulation but 17-OHP and testosterone inhibition following $100 \mu \mathrm{M}$ DzBP or DEHP treatments) is associated with up-regulation of mitochondrial P450SCC but down-regulation of StAR and CYP17 $\alpha$ (Figure 4, see SI Table S9), which are the two genes just up-stream and down-stream of P450SCC (Figure 5). All the data implies that CYP17 $\alpha$ initiates the anti-androgenic effect in the investigated steroidogenesis pathway.

\section{Discussion}

We observed that both extracellular esterase and the CAR-regulated intracellular carboxylesterase can catalyse the hydrolysis of phthalate diesters, and that these processes are closely associated with their endocrine disrupting effects (Figure 5).

Meanwhile, phthalate diesters' hydrolysis and endocrine disrupting potency depends on their alkyl side-chain structure. DBP elicits hydrolysis-related steroidogenic modulation, in which the anti-androgenic effect of this diester reverses into an androgenic effect of monoester MBP at $100 \mu \mathrm{M}$. Monoesters and diesters exhibit apparently differing endocrine disrupting effects, and these results were tested in both the transcriptional alteration of androgen synthesis pathway genes and fold-changes in three steroids. Our work indicates that the hydrolysis of phthalate diesters into monoesters is generally an anti-androgenic reversal process.

\subsection{Phthalate diesters' hydrolysis in MLTC-1 cells}

It is well-documented that phthalate diesters can be quickly hydrolysed into monoesters in mammals by some non-specific esterases and lipases in the intestines 
and parenchyma. Some long-chain monoesters can be in further metabolized via $\beta$-oxidation and then formed the hydrophilic glucuronide conjugates, which are easily excreted in urine (Calafat et al. 2006; Frederiksen et al. 2007). Our in vitro tests show that the hydrolysis of phthalate diesters by extracellular esterase is side-chain structure specific. Like many other xenobiotics, intracellular metabolism of phthalate diesters can be regulated by CAR. Intracellularly, diesters can bind to CAR with a higher potency than monoesters (Zhang et al. 2015). Our observations are further supported by the fact that the binding activity affects down-stream carboxylesterases (Ces) activity. Ces can been found in various tissues, such as the small intestine, colon, kidney, liver, heart, brain and testis (Xu et al. 2002). It has been recognized to play an important role in biotransformation of ester- and amide-containing compounds for detoxification and/or activation of a variety of xenobiotics and pharmacologics (Satoh and Hosokawa 2006). A previous study suggests that mRNA expression of Ces in mouse liver, duodenum and lung tissues are regulated by intracellular CAR (Jones et al. 2013); we found Ces expression to be also regulated by CAR in MLTC-1 cells. In using the CAR regulation of Ces, we tested whether testicular MLTC-1 cells can hydrolyse phthalate diesters into monoesters. Similar to liver microsomes (Hanioka et al. 2012), MLTC-1 cells exhibit high activity towards short-chain phthalates (i.e., DMP, DEP and DBP), while activity towards DEHP is marginal. Surprisingly aromatic chain DBzP cannot be hydrolysed into MBzP in MLTC-1 cells at any exposure. Therefore, the hydrolysis of diesters by intracellular Ces are also alkyl chain specific. This may be due to the bulky side-chains of DEHP and DBzP 
protecting their ester bonds, resulting in poor bioavailability. The molecular specific hydrolysis agrees with in vitro liver microsomes tests and an in vivo kinetics study (Hanioka et al. 2012; Harris et al. 2016). In addition, the hydrolysis rates to high-dose diesters $(100 \mu \mathrm{M})$ are lower than to low-dose $(10 \mu \mathrm{M})$, so it is suspected that the process is not inducible; this is inconsistent with this notion that CAR regulation is constitutive but inducible.

\subsection{Phthalate diesters and monoesters associated with distinct endocrine effects}

Regarding phthalate structures associated with endocrine disrupting activities, there remains controversy. Many phthalate diesters are implied to be less toxic towards testosterone generation than their monoester counterparts, e.g., DEHP and DBP versus MEHP and MBP (Clewell et al. 2010; Sjöberg et al. 1986). However, a previous study also reports that DBP is more toxic than MBP in its inhibition of placental steroids (Xu et al. 2016). Although all previous studies (Barlow et al. 2003;

Clewell et al. 2010; Parks et al. 2000) support the notion of phthalates inducing endocrine disrupting activity in testicular cells, the present work uncovers the molecular specific differences between diesters and monoesters: diesters at low-dose $(10 \mu \mathrm{M})$ can slightly induce progesterone, ASD and testosterone secretion, and these trends are similar to $100 \mu \mathrm{M}$ monoesters. When the intracellular diesters' hydrolysis is inhibited, testosterone secretion is significantly decreased (Figure 3). Therefore, monoesters or the hydrolysis of diesters to monoesters is associated with androgenic effects. While diesters exhibit an apparent anti-androgenic effect at high-dose (100 $\mu \mathrm{M})$ exposure levels, it is because the major components of these phthalates are 
diesters rather than monoesters in the cells. The individual exposure experiments also confirmed that the high-dose diesters have more potency in inhibiting steroid hormone secretion than monoesters (Figure 4). Given the environmentally-relevant exposures dose, phthalate diesters can be quickly hydrolysed into monoesters forms in the human body by a more comprehensive set of hydrolytic enzymes than in vitro tests, their activity would be reversed from anti-androgenic to androgenic.

\subsection{Hydrolysis attenuates phthalate diesters' anti-androgenic effects in MLTC-1 cells}

Androgen biosynthesis in Leydig cells involves the conversion from cholesterol, via a series of intermediates, to the end-product, testosterone. The reactions are catalysed by enzymes of SR-B1, StAR, P450SCC, Cyp17 $\alpha, 3 \beta-H S D$ and 17 $\beta$-HSD

(Ha et al. 2016) (Figure 5). Extracellular hydrolysis of phthalates diesters to monoesters can attenuate their apparently anti-androgenic effects both at gene transcriptional and metabolic levels. Impacts on all investigated steroidogenic genes were partly to completely reduced, and the production of 17-OHP and testosterone recovered whilst for ASD, there is an increase in comparison to the control (Figure 2). In human and rodent steroidogenic tissues, SR-B1 transports the cholesteryl esters into cells which is subsequently converted into pregnenolone by P450SCC in the inner mitochondrial membrane and, finally into progesterone via Cyp $17 \alpha$ and $3 \beta$-HSD catalysis (Azhar and Reaven 2002). Most notable observations are the initial up-/down-regulation of $S R-B 1$, which may be mostly responsible for the distinct modes of endocrine disrupting effects between phthalate diesters and monoesters 
(Figure 4). The decrease in testosterone with $S R-B 1$ and StAR gene expression post-exposure of rats and mouse to phthalates has been reported (Aydoğan and Barlas 2015; Barlow et al. 2003; Wang et al. 2017). We propose that phthalate monoesters have more potential than their diester counterparts to initiate $S R-B 1$ up-regulation for steroidogenesis; its inhibition would be associated with the down-regulation of Cyp $17 \alpha$ and StAR, which are the down- and up-stream genes of the mitochondrial P450SCC. SR-B1 lead the up-regulation of all steroidogenesis genes such as P450SCC and Cyp17 $\alpha$ and their expression together with increased ASD may imply the androgenic responses to monoesters' exposure or diesters' hydrolysis (Figure 4). While high-dose diesters (Figure 1) or the inhibition of their hydrolysis (Figure 3,5)

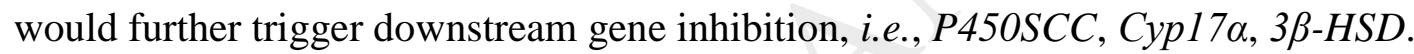
Interestingly, in response to low- to high-dose stimulation, $C y p 17 \alpha$ expression altered dramatically and led to down-regulated gene expression, which may imply a complex the steroidogenic regulation. $C Y P 17 \alpha$ is at a critical crossroad point in the pathways of steroid hormone biosynthesis (Gilep et al., 2011), and the enzymes of P450SCC and StAR are considered as rate-limiting in the steroid hormone synthesis pathway (Hasegawa et al., 2000, Miller, 1998). This information may also imply Cyp17 $\alpha$ initiates down-regulation of steroidogenesis via its feedback to P450SCC and StAR, in which inhibited StAR may initially respond to down-regulated Cyp $17 \alpha$, with steroids partly decreased (examples are $100 \mu \mathrm{M}$ DBzP and DEHP). Then P450SCC is subsequently inhibited and steroids are completely inhibited (e.g., $100 \mu \mathrm{M} \mathrm{DBP})$. The mitochondrial-related backward regulation needs to be tested in further study. 
Structure-dependent activity of phthalates has also been documented for other steroidogenic enzymes, such as inhibition of $17 \beta$-hydroxysteroid dehydrogenase 3 (Yuan et al. 2012), 11ß-hydroxysteroid dehydrogenase 2 (Zhao et al. 2010) and 3ß-hydroxysteroid dehydrogenase 1 CYP19A1(Xu et al. 2016). Our results indicate that diesters have more potency on steroidogenic gene expression, which is consistent with previous reports that diesters are inhibitors of CYP19A1 and CYP2B, while monoesters have no or little inhibition (Ozaki et al. 2016; Xu et al. 2016). The diesters and monoesters appear to exert different effects on the activity of human hormone receptors (AR and ER). The phthalates either stimulated or inhibited ER $\alpha$ and ER $\beta$ activity and inhibited AR activity, whereas the phthalate metabolites had no impact (Engel et al., 2017). Similar results also found in CAR-binding activities studies, due to phthalate monoester having only one side-chain to interact with the CAR active pocket, their binding activities are lower than those of their corresponding phthalate diesters (Zhang et al., 2015). Interestingly, the androgenic potency of phthalate diesters are found to increase with increasing carbon number of the linear alkyl chains from DMP to DBP, while anti-androgenic potency decreases from DBP to long/bulky DBzP and DEHP (Figure 4). The U-shaped endocrine disrupting effects of phthalate are in good agreement with the conformations of the phthalates upon binding to constitutive androstane receptor (Zhang et al. 2015).

\section{Conclusion}

The present study shows that phthalate diesters have side-chains structurally dependent on hydrolysis and steroidogenic effects in MLTC-1 Leydig cells. The 
results indicate that DBP seems to elicit hydrolysis-related steroidogenesis modulation, in which the complete anti-androgenic effect of diester DBP reverses into a androgenic effect when it is hydrolysed into monoester MBP at $100 \mu \mathrm{M}$ in Leydig cells. When compared to DBP, the anti-androgenic potency of DBzP and DEHP may be lower; the generation of androgens cannot be completely inhibited (ASD is stimulated) by DBzP and DEHP in Leydig cells even when treated with $100 \mu \mathrm{M}$, but the down-regulation tendency has been observed. DEP and DMP and all the investigated monoester phthalates only exhibit androgenic effects at the treatment dose ranges. Considering the ready metabolism of the investigated phthalate diesters in the human body, the observed toxicology improves our understanding of the environmentally-relevant phthalates' reproduction risk in adult males.

\section{Funding information}

This work is financially supported by the National Nature Science Foundation of China (NSFC-21307126; 21177123; 21407144), the Crossing-Group Project of

KLUEH (KLUEH-201802), the Knowledge Innovation Program of the Chinese Academy of Sciences (IUEQN201506), Nature Science Foundation of Fujian Province (2017J01027), and Zhoushan Municipal Medical and Health Foundation (2016A18).

\section{Conflict of interest statement}

The authors declare that there are no conflicts of interest. 


\section{References}

1. Aydoğan, A.M., Barlas, N., 2015. Influence of in utero di-n-hexyl phthalate and dicyclohexyl phthalate on fetal testicular development in rats. Toxicol. Lett. 233, $125-137$

2. Azhar, S., Reaven, E., 2002. Scavenger receptor class BI and selective cholesteryl ester uptake: partners in the regulation of steroidogenesis. Mol. Cell Endocrinol. $195,1-26$

3. Barlow, N.J., Phillips, S.L., Wallace, D.G., Sar, M., Gaido, K.W., Foster, P.M., 2003. Quantitative changes in gene expression in fetal rat testes following exposure to di(n-butyl) phthalate. Toxicol. Sci. 73, 431-441

4. Blanchard, O., Glorennec, P., Mercier, F., Bonvallot, N., Chevrier, C., Ramalho, O., Mandin, C., Bot, B.L., 2014. Semivolatile organic compounds in indoor air and settled dust in 30 French dwellings. Environ. Sci. Technol. 48, 3959-3969.

5. Bochud, M., Jenny-Burri, J., Pruijm, M., Ponte, B., Guessous, I., Ehret, G., Petrovic, D., Dudler, V., Haldimann, M., Escher, G., Dick, B., Mohaupt, M., Paccaud, F., Burnier, M., Péchère-Bertschi, A., Martin, P.Y., Vogt, B., Ackermann, D., 2018. Urinary Cadmium Excretion Is Associated With Increased Synthesis of Cortico- and Sex Steroids in a Population Study. J. Clin. Endocrinol. Metab. 103, 748-758.

6. Calafat, A.M., Ye, X., Silva, M.J., Kuklenyik, Z., Needham, L.L., 2006. Human exposure assessment to environmental chemicals using biomonitoring. Int. J. Androl. 29, 166-171.

7. Clewell, R.A., Campbell, J.L., Ross, S.M., Gaido, K.W., Clewell, H.J., Andersen, M.E., 2010. Assessing the relevance of in vitro measures of phthalate inhibition of steroidogenesis for in vivo response. Toxicol. In Vitro 24, 327-334.

8. Engel, A., Buhrke, T., Imber, F., Jessel, S., Seidel, A., Völkel, W., Lampen, A., 2017. Agonistic and antagonistic effects of phthalates and their urinary metabolites on the steroid hormone receptors ER $\alpha$, ER $\beta$, and AR. Toxicol. Lett. $277,54-63$.

9. Fernández, M.A., Gómara, B., González, M.J., 2012. Occurrence of Phthalates 
and Their Metabolites in the Environment and Human Health Implications. Springer Berlin Heidelberg.

10. Frederiksen, H., Skakkebaek, N.E., Andersson, A.M., 2007. Metabolism of phthalates in humans. Mol. Nutr. Food Res. 51, 899-911.

11. Ha, M., Guan, X., Wei, L., Li, P., Yang, M., Liu, C., 2016. Di-(2-ethylhexyl) phthalate inhibits testosterone level through disturbed hypothalamic-pituitary-testis axis and ERK-mediated $5 \alpha$-Reductase 2. Sci. Total. Environ. 563-564, 566-575.

12. Hanioka, N., Takahara, Y., Takahara, Y., Tanaka-Kagawa, T., Jinno, H., Narimatsu, S., 2012. Hydrolysis of di-n-butyl phthalate, butylbenzyl phthalate and di(2-ethylhexyl) phthalate in human liver microsomes. Chemosphere 89, $1112-1117$.

13. Harris, S., Wegner, S., Hong, S.W., Faustman, E.M., 2016. Phthalate metabolism and kinetics in an in vitro model of testis development. Toxicol. In Vitro 32, $123-131$

14. Hauser, R., Calafat, A.M., 2005. Phthalates and human health. Occup. Environ. Med. 62, 806-818.

15. Howdeshell, K., Wilson, V., Furr, J., Lambright, C.R., Rider, C.V., Blystone, C.R., Hotchkiss, A.K., Gray, LE. Jr., 2008. A mixture of five phthalate esters inhibits fetal testicular testosterone production in the sprague-dawley rat in a cumulative, dose-additive manner. Toxicol. Sci. 105:153-165.

16. Jones, R.D., Taylor, A.M., Tong, E.Y., Repa, J.J., 2013. Carboxylesterases are uniquely expressed among tissues and regulated by nuclear hormone receptors in the mouse. Drug Metab. Dispos. 41, 40-49.

17. Katsikantami, I., Sifakis, S., Tzatzarakis, M.N., Vakonaki, E., Kalantzi, O.I., Tsatsakis, A.M., Rizos, A.K., 2016. A global assessment of phthalates burden and related links to health effects. Environ. Int. 97, 212-236.

18. Koch, H.M., Rossbach, B., Drexler, H., Angerer, J., 2003. Internal exposure of the general population to DEHP and other phthalates--determination of secondary and primary phthalate monoester metabolites in urine. Environ. Res. 93, 177-185. 
19. Liu, L., Bao, H., Liu, F., Zhang, J., Shen, H., 2012. Phthalates exposure of Chinese reproductive age couples and its effect on male semen quality, a primary study. Environ. Int. 42, 78-83.

20. Mankidy, R., Wiseman, S., Ma, H., Giesy, J.P., 2013. Biological impact of phthalates. Toxicol. Lett. 217, 50-58.

21. Moore, R.W., Rudy, T.A., Lin, T.M., Ko, K., Peterson, R.E., 2001. Abnormalities of sexual development in male rats with in utero and lactational exposure to the antiandrogenic plasticizer Di(2-ethylhexyl) phthalate. Environ. Health. Perspect. 109, 229-237.

22. Ozaki, H., Sugihara, K., Watanabe, Y., Ohta, S., Kitamura, S., 2016. Cytochrome P450-inhibitory activity of parabens and phthalates used in consumer products. $\mathbf{J}$ Toxicol. Sci. 41, 551-560.

23. Park, S., Kim, B..N, Cho, S.C., Kim, Y., Kim, J.W., Lee, J.Y., Hong, S.B., Shin, M.S., Yoo, H.J., Im, H1., Cheong, J.H., Han, D.H., 2014. Association between urine phthalate levels and poor attentional performance in children with attention-deficit hyperactivity disorder with evidence of dopamine gene-phthalate interaction. Int. J. Environ. Res. Public. Health 11, 6743-6756.

24. Parks, L.G., Ostby, J.S., Lambright, C.R., Abbott, B.D., Klinefelter, G.R., Barlow, N.J., Gra,y LE. Jr., 2000. The plasticizer diethylhexyl phthalate induces malformations by decreasing fetal testosterone synthesis during sexual differentiation in the male rat. Toxicol. Sci. 58:339-349.

25. Satoh, T., Hosokawa, M., 2006. Structure, function and regulation of carboxylesterases. Chemico-Biological Interactions 162, 195-211.

26. Shea KM. (2003). Pediatric exposure and potential toxicity of phthalate plasticizers. Pediatrics 111, 1467-1474.

27. Sjöberg, P., Bondesson, U., Gray, T.J., Plöen, L., 1986. Effects of Di-(2ethylhexyl) Phthalate and Five of its Metabolites on Rat Testis in Vivo and in in Vitro. Acta. Pharmacol. Toxicol. 58(3):225-233.

28. Stein, T.P., Schluter,. MD., Steer, R.A., Ming, X., 2013. Autism and phthalate 
metabolite glucuronidation. J. Autism Dev. Disord. 43, 2677-2685.

29. Svechnikov, K., Savchuk, I., Morvan, M.L., Antignac, J.P., Le Bizec, B., Soder, O., 2016. Phthalates Exert Multiple Effects on Leydig Cell Steroidogenesis. Horm. Res. Paediatr. 86, 253-263.

30. Swan, S.H., 2008. Environmental phthalate exposure in relation to reproductive outcomes and other health endpoints in humans. Environ. Res. 108, 177-184.

31. Tian, M.P., Liu, L.P., Wang, H., Wang, X.F., Martin, F.L., Zhang, J., Huang, Q.Y., Shen, H.Q., 2018. Phthalates Induce Androgenic Effects at Exposure Levels That Can Be Environmentally Relevant in Humans. Environ. Sci. Technol. Lett. 5, 232-236.

32. Wang, X., Sheng, N., Cu, R., Zhang, H., Wang, J., Dai, J., 2017. Gestational and lactational exposure to di-isobutyl phthalate via diet in maternal mice decreases testosterone levels in male offspring. Chemosphere 172, 260-267.

33. Xu, G., Zhang, W., Ma, M.K., Mcleod, H.L., 2002. Human carboxylesterase 2 is commonly expressed in tumor tissue and is correlated with activation of irinotecan. Clin. Cancer Res. 8, 2605-2611.

34. Xu, R.A., Mao, B., Li, S., , Liu, J., Li, X., Li, H., Su, Y., Hu, G., Lian, Q.Q., Ge, R.S., 2016. Structure-activity relationships of phthalates in inhibition of human placental 3 $\beta$-hydroxysteroid dehydrogenase 1 and aromatase. Reprod. Toxicol. 61, 151-161.

35. Yuan, K., Zhao, B., Li, X.W., Hu, G.X., Su, Y,, Chu, Y., Akingbemi, B.T., Lian, Q.Q., Ge, R.S., 2012. Effects of phthalates on 3 $\beta$-hydroxysteroid dehydrogenase and 17ß-hydroxysteroid dehydrogenase 3 activities in human and rat testes. Chem. Biol. Interact. 195, 180-188.

36. Zhang, H., Zhang, Z.B., Nakanishi, T., Wan, Y., Hiromori, Y., Nagase, H., Hu, J., 2015. Structure-Dependent Activity of Phthalate Esters and Phthalate Monoesters Binding to Human Constitutive Androstane Receptor. Chem. Res. Toxicol. 28, 1196-1204.

37. Zhao, B., Chu, Y., Huang, Y., Hardy, D.O., Lin, S., Ge, R.S., 2010.

Structure-dependent inhibition of human and rat 11beta-hydroxysteroid 
dehydrogenase 2 activities by phthalates. Chem. Biol. Interact. 183, 79-84.

38. Zhu, K.Y., He, F., 2000. Elevated Esterases Exhibiting Arylesterase-like Characteristics in an Organophosphate-Resistant Clone of the Greenbug, Schizaphis graminum (Homoptera: Aphididae). Pestic Biochem. Physiol. 67, 155-167. 


\section{Figure Legends}

Figure 1. Phthalates ester bonds associated with phthalates endocrine disruption. Phthalate diesters metabolism profile with mixture exposure in MLTC-1 cell (A), steroidogenesis pathway gene expression (B-C) and steroid hormones (17-OHP, ASD and testosterone) secretion response to diesters or monoesters mixed exposure (D-E). M: equimolar phthalate monoester mixtures (MMP, MEP, MBP, MBzP or MEHP in mixture) and D: equimolar phthalate diester mixtures (DMP, DEP, DBP, DBzP or DEHP in mixture).

Data presented as Mean \pm SEM is derived from three independent experiments. Heat maps showing changes in gene expression for steroidogenesis pathway gene after phthalate exposure, red colour represents up-regulation and green colour represents down-regulation. Asterisks indicate statistically significant differences, $* p<0.05$ and $* * p<0.01$ in comparison to the vehicle.

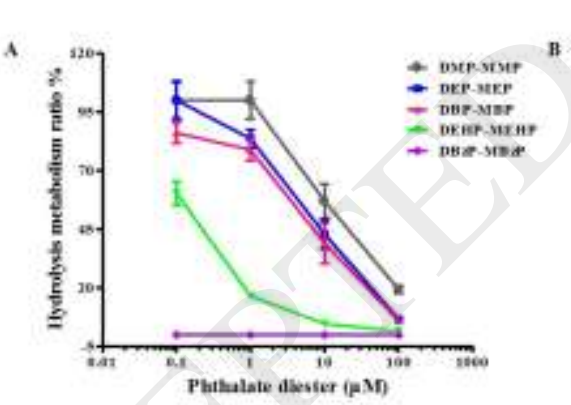

17-OH Progesterone

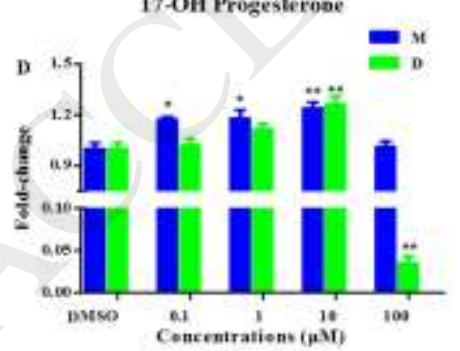

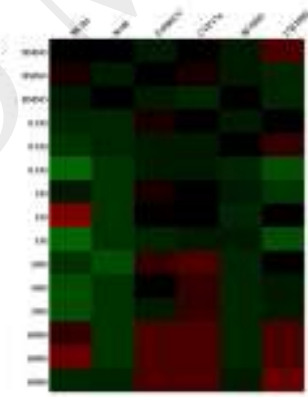

Androsteredione

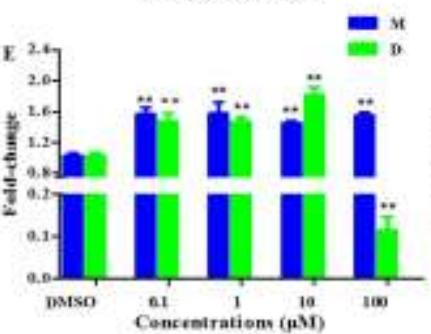

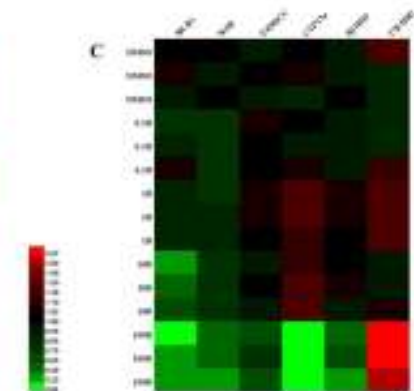

Testosterone

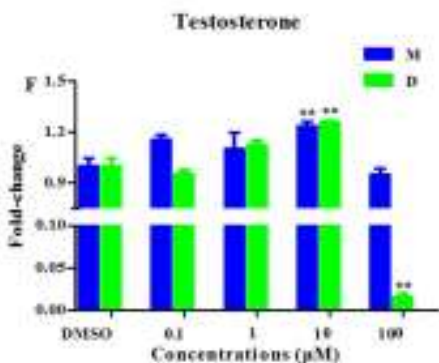

Figure 2. The extracellular esterase enhances phthalate diesters' hydrolysis and attenuates their anti-androgenic effects. 
The extracellular esterase facilitated phthalate diesters hydrolysis (A) can attenuate their anti-androgenic effects by altering the steroidogenesis pathway gene expression (B) and reversing androgen secretions (C-E) in the MLTC-1 cells.

$100 \mathrm{M}: 100 \mu \mathrm{M}$ of equimolar phthalate monoester mixtures (MMP, MEP, MBP, MBzP or MEHP in mixture); 100D: $100 \mu \mathrm{M}$ of equimolar phthalate diester mixtures (DMP, DEP, DBP, DBzP or DEHP in mixture); 100D+: the addition of esterase in culture matrix to $100 \mathrm{D}$ group to facilitate phthalate diester hydrolysis.

Data presented as mean \pm SEM of the three independent experiments. Heat maps showing changes in gene expression for steroidogenesis pathway gene after phthalate exposure, red colour represents up-regulation and green colour represents down-regulation. Asterisks indicate statistically significant differences, ${ }^{*} p<0.05$ and $* * p<0.01$.
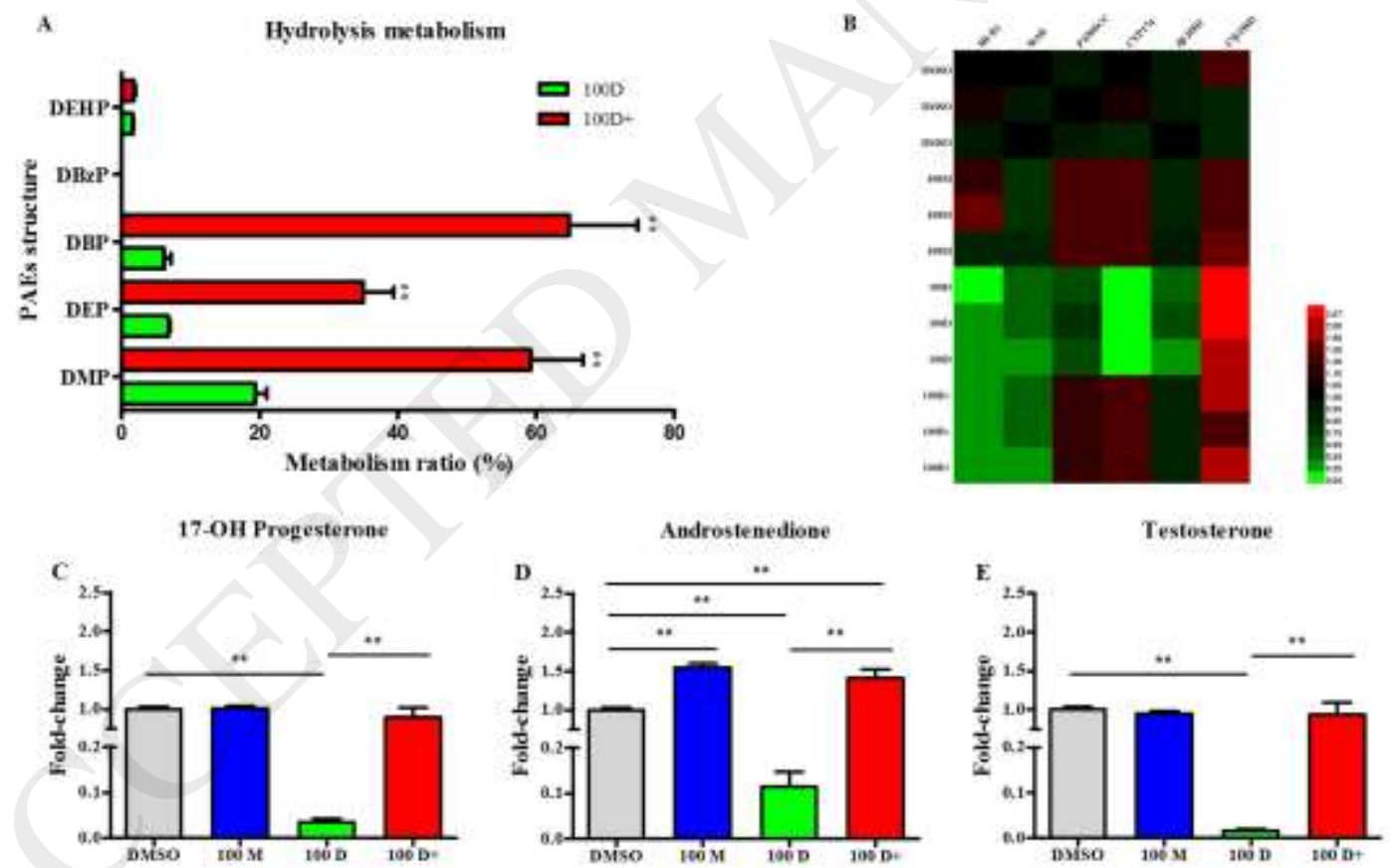

Figure 3. Inhibited intracellular carboxylesterase associated with reduced hydrolysis and enhanced activity of phthalate diesters.

PK 11195 inhibits intracellular carboxylesterase gene expression (A) and enzyme 
activity (B) then reduced diesters hydrolysis (C) and decreased steroidogenesis pathway genes expression (D) and androgen secretion (E-G).

10D: $10 \mu \mathrm{M}$ of equimolar DMP, DEP, DBP, DEHP and DBzP mixture, respectively. PK 11195: $5 \mu \mathrm{M}$

1-(2-Chlorophenyl)-N-methyl-N-(1-methylpropyl)-3-isoquinolinecarboxamide, 10D+PK 11195: $10 \mu \mathrm{M}$ phthalate diesters mixture plus with $5 \mu \mathrm{M}$ PK 11195.

Data are presented as Mean \pm SEM of three independent experiments. Heat maps showing changes in gene expression for steroidogenesis pathway gene after phthalate exposure, red colour represents up-regulation and green colour represents down-regulation. Asterisks indicate statistically significant differences, ${ }^{*} p<0.05$ and $* * p<0.01$.
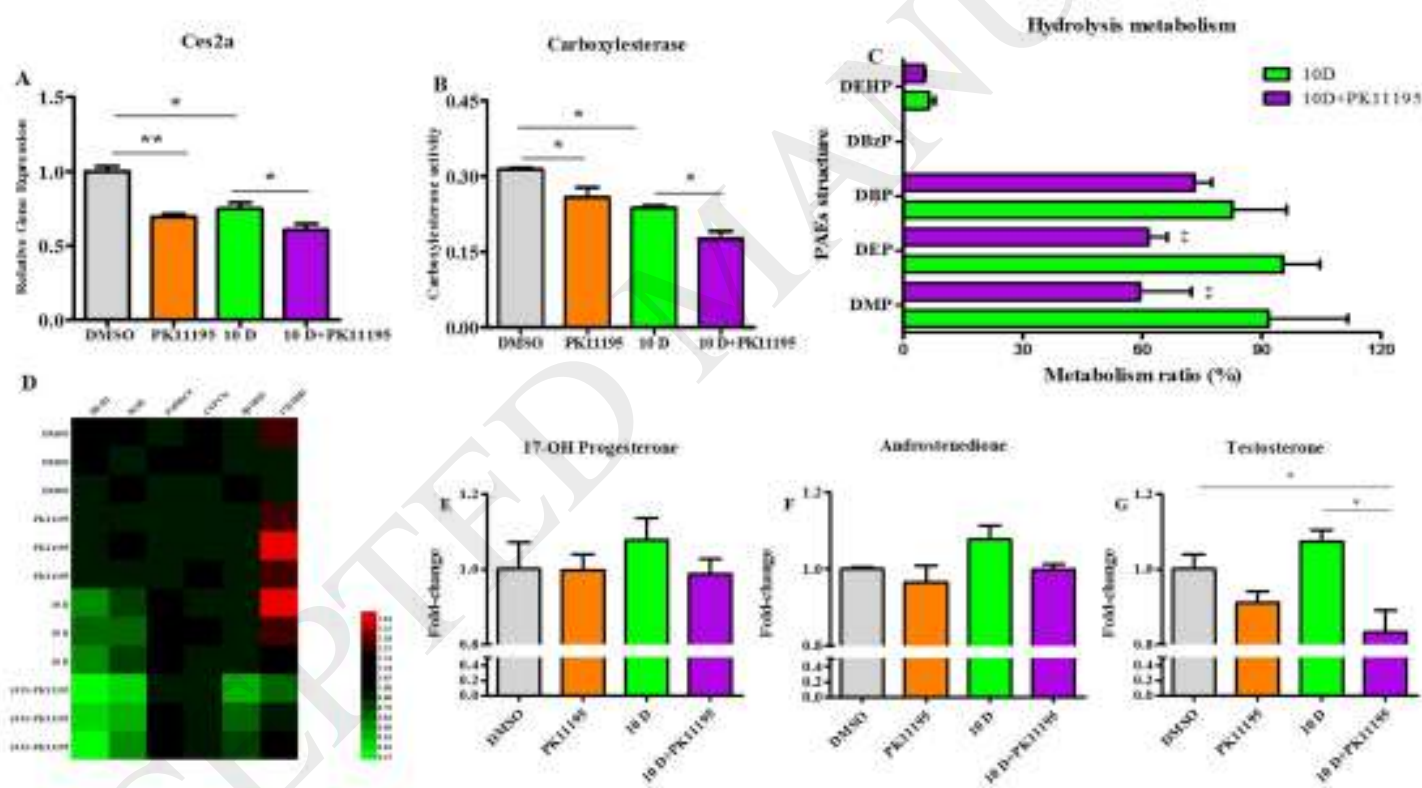

Figure 4. Different endocrine disrupting effects between phthalate diesters (A-D) and monoesters (E-H) individual exposure.

Data are presented as Mean \pm SEM of three independent experiments. Heat maps showing changes in gene expression for steroidogenesis pathway gene after phthalate exposure, red colour represents up-regulation and green colour represents down-regulation. Compared with DMSO vehicle group, asterisks indicate statistically significant differences, ${ }^{*} p<0.05$ and ${ }^{*} * p<0.01$. 

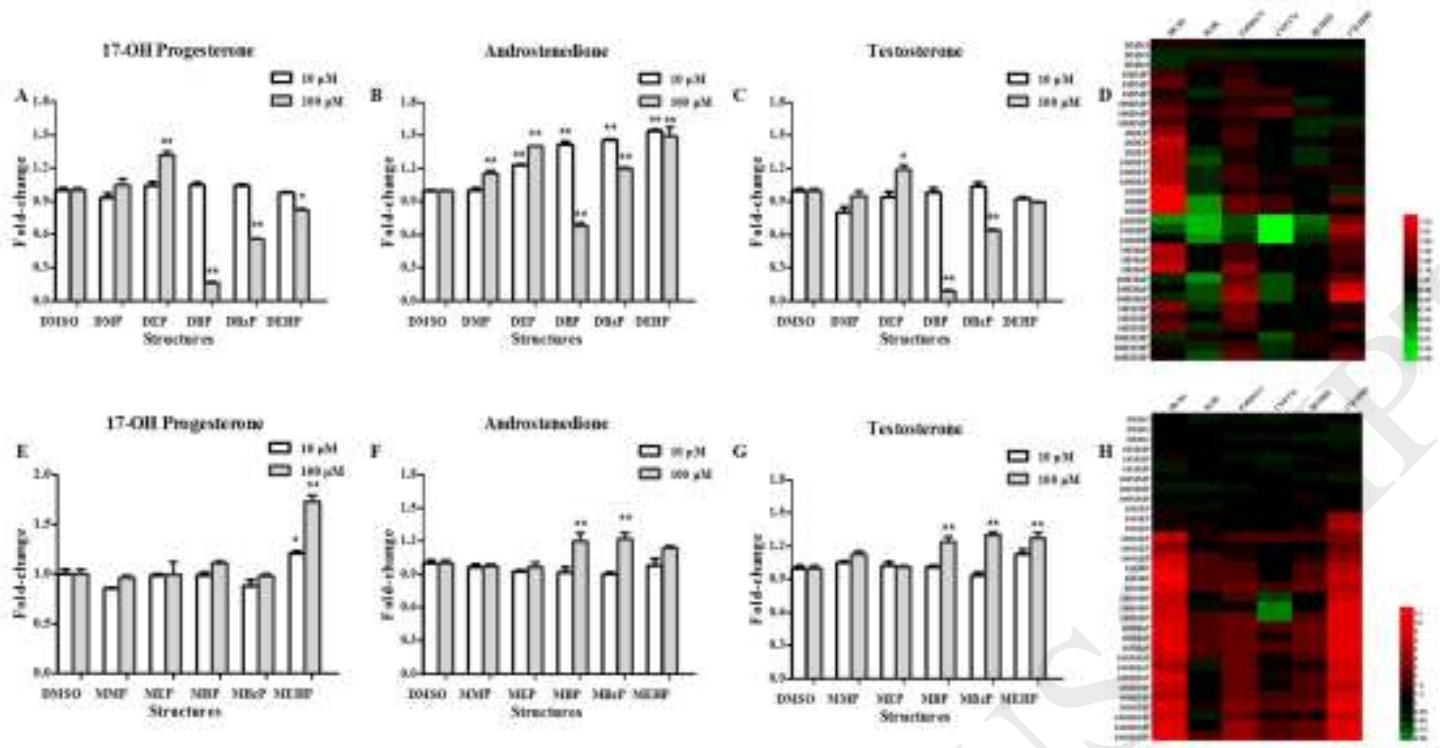

Figure 5. Phthalate hydrolysis metabolism associated with steroidogenesis effects in MLTC-1 Leydig cells.

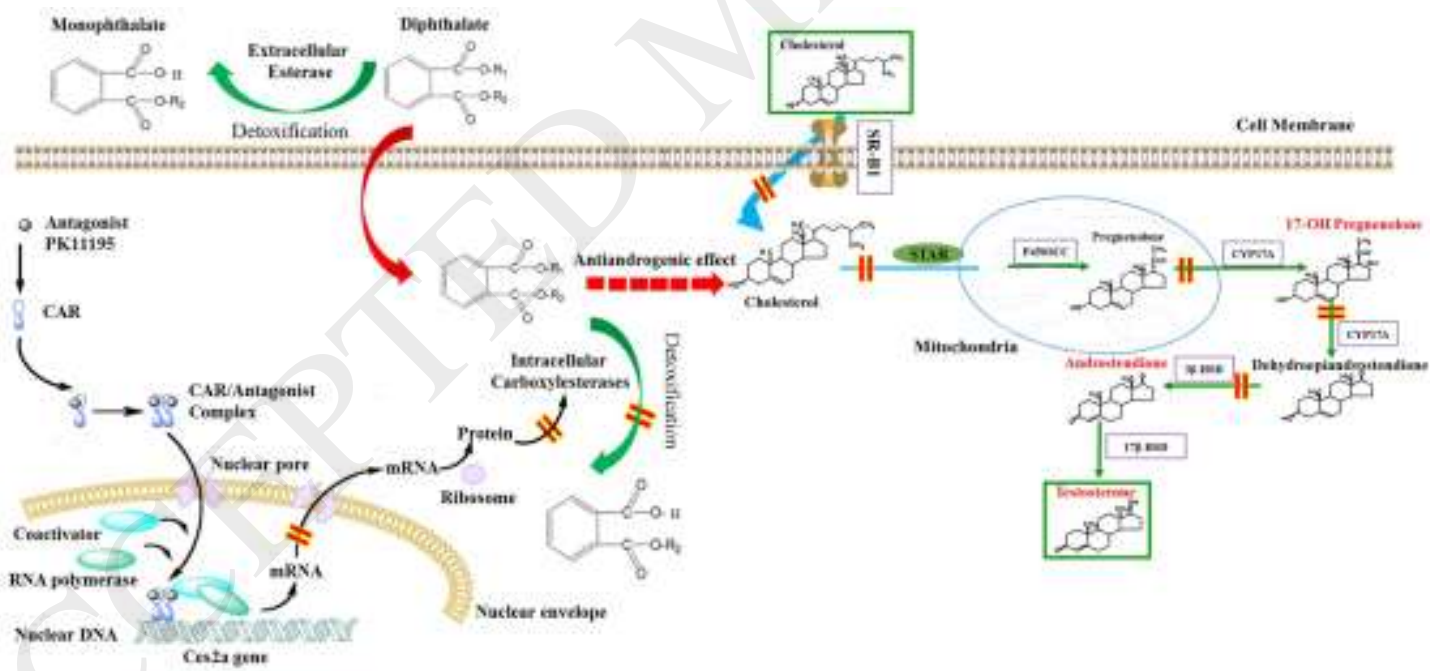

II Inhibition effects 